\title{
Espaços de produção da leishmaniose tegumentar americana no estado do Rio de Janeiro, Brasil
}

\author{
Spaces of production of cutaneous leishmaniasis in the state \\ of Rio de Janeiro, Brazil
}

Valdenir Bandeira Soares (https://orcid.org/0000-0002-4800-2940) ${ }^{1}$

Paulo Chasgastelles Sabroza (https://orcid.org/0000-0001-8235-0562) ${ }^{1}$

Waldemir Paixão Vargas (https://orcid.org/0000-0003-3692-7185) ${ }^{1}$

Reinaldo Souza-Santos (https://orcid.org/0000-0003-2387-6999) ${ }^{1}$

Ana Cecília de Oliveira Valdés (https://orcid.org/0000-0002-1158-5476) ${ }^{1}$

Andréa Sobral (https://orcid.org/0000-0003-0552-771X) ${ }^{1}$

${ }^{1}$ Escola Nacional de Saúde Pública, Fiocruz. R. Leopoldo Bulhões 1480, Manguinhos. 21041-210 Rio de Janeiro RJ Brasil. valdenir@ensp.fiocruz.br

\begin{abstract}
The scope of this research was to identify and characterize spatial units of epidemiological relevance in the state of Rio de Janeiro, through the highest concentrations of cutaneous leishmaniasis (CL) from 1980 to 2012, considering the geographical aspects. SUCAM, FUNASA and SINAN databases were consulted. A method of adjustment of spatially referenced data for demarcation of the regions with the highest concentrations of cases called circuits and poles was applied. These were superimposed on the socioenvironmental indicator maps. Of the total cases registered in the period, $87 \%$ occurred in the municipalities located in the resulting circuits and poles. The variations in the occurrence of cases in the different circuits and poles were not related to the socioenvironmental indicators. The identification of the circuits and poles can subsidize the state CL program of the prioritization of strategies of prevention and control actions and the optimization of the resources of the program. These regions, which are more stable than the localities, allow surveillance and control operations in locations with many cases and in other locations in the identified risk area, because they have the same characteristics as those already affected.

Key words Cutaneous leishmaniasis, Epidemiology, Geographic risk location, Geographical mapping, Epidemiological surveillance
\end{abstract}

Resumo $O$ objetivo foi identificar e caracterizar unidades espaciais de relevância epidemiológica no estado do Rio de Janeiro, por meio das maiores concentrações de casos de leishmaniose tegumentar (LT) no período de 1980 a 2012, considerando os conceitos da geografia. Utilizou-se bancos de dados da SUCAM, FUNASA e SINAN. Foi aplicado um método de ajustamento de dados espacialmente referenciados para delimitação das regiões com as maiores concentrações de densidades de casos chamadas circuitos e polos. Estes foram sobrepostos aos mapas de indicadores socioambientais. Do total de casos registrados no período, 87\% ocorreram nos municípios localizados nos circuitos e polos resultantes. As variações na ocorrência de casos nos diferentes circuitos $e$ polos não tiveram relação com os indicadores socioambientais. A identificação dos circuitos e polos pode subsidiar o programa estadual da LT para a priorização de estratégias de ações de prevenção e controle e a otimização dos recursos do programa. Essas regiões, mais estáveis que as localidades, permitem operações de vigilância e controle nas localidades com muitos casos e nas demais da área de risco identificada, por terem as mesmas características daquelas já afetadas.

Palavras-chave Leishmaniose cutânea, Epidemiologia. Localização geográfica de risco, Mapeamento geográfico, Vigilância epidemiológica 


\section{Introdução}

As transformações nos processos de produção, em decorrência da globalização e da consolidação do modelo capitalista técnico-científico tem resultado em crescimento econômico e na expansão de um contingente populacional integrado nas sociedades de modo desigual e incompleto, configurando um circuito espacial de produção inferior ${ }^{1}$.

Desemprego, desinserção territorial, falta de perspectiva de ascensão social e restrição de acesso aos recursos de seguridade social se combinam com a exposição a um nível crescente de informação, indução de novos padrões de consumo e perspectivas de elaboração de novas estratégias de reprodução, resultando no aumento da economia informal ${ }^{1}$ na expansão das periferias urbanas $^{2}$ e no incremento da mobilidade destes grupos sociais desterritorializados, amplificando as possibilidades de circulação de parasitas ${ }^{3}$.

Nas últimas décadas, mudanças no comportamento de endemias vêm sendo observadas, possibilitando sua disseminação por meio de redes de circulação dos parasitas, viabilizadas pela integração territorial, maior mobilidade das populações e construção de novas condições de receptividade, assegurando sua difusão para outras áreas e grupos sociais ${ }^{4,5}$.

No Brasil, desde a metade da década de 1970, tem sido registrado um aumento progressivo do número de registros da leishmaniose tegumentar $(\mathrm{LT})^{6,7}$ e de sua área de ocorrência nas diversas regiões do país ${ }^{8-10}$. Na década de 1980 foram registrados e investigados surtos por todo o estado do RJ, sempre com as mesmas características locais, que ressaltavam a importância do domicílio como fator de risco na transmissão da LT, mas com outra dinâmica espaço-temporal ${ }^{11}$.

Em 1995, Soares et al. identificaram e caracterizaram o que chamaram de Circuitos de produção da LT no estado do RJ. Os circuitos foram definidos pela elevada concentração de casos com diferentes gradientes, podendo se superpor a mais de um município ${ }^{12}$.

Nesse contexto, Soares et al., em 2017, apontaram a região da Baía da Ilha Grande (Angra dos Reis e Parati) como importante polo produtor da LT, o que permitiu a identificação de áreas de risco da doença em escala intramunicipal denominadas Unidades de Vigilância Local (UVL) ${ }^{13}$.

O objetivo do estudo foi identificar e caracterizar unidades espaciais de relevância epidemiológica no estado do RJ, por meio das maiores concentrações de casos de LT no período de 1980 a 2012, considerando os conceitos da geografia.

\section{Metodologia}

O estado do RJ, no sudeste do Brasil, é composto por 92 municípios (Figura 1). Segundo o Instituto Brasileiro de Geografia e Estatística (IBGE) de 2010, sua população foi de 15.989 .929 habitantes, sendo que 6.320.446 destes residiam no município do Rio de Janeiro. A densidade populacional no estado é muito heterogênea, podendo variar de 12,67 em Santa Maria Madalena na região Serrana, para 13.030,48 habitantes por $\mathrm{km}^{2}$ em São João de Meriti na região metropolitana.

Foi organizado um banco de registros de casos autóctones de LT por municípios de residência de 1990 a 2012, obtidos da seguinte forma: de 1990 a 1995 foi utilizado o banco da Superintendência de Campanhas de Saúde Pública (SUCAM), de 1996 a 1999 foi usado um banco da Fundação Nacional de Saúde (FUNASA) e de 2000 a 2012 adotou-se o Sistema de Informação de Agravos de Notificação (SINAN).

O banco foi organizado com as seguintes variáveis: código do IBGE do município de residência, nome do município de residência, ano de diagnóstico, sexo, idade e fonte da informação. Para o período de 2000 a 2012 foram usadas, além dessas, outras informações contidas no SINAN, como forma clínica (mucosa), critério de confirmação (diagnóstico laboratorial) e evolução do caso (cura).

Para identificar as variações na ocorrência de casos de LT no estado, elaborou-se um gráfico com a distribuição anual dos casos de 1990 a 2012. Para atenuar essas flutuações irregulares e melhor observar as tendências foi inserida uma curva por meio das médias móveis de três anos.

As áreas de maior concentração de casos no estado foram identificadas considerando duas categorias de análise relativas à distribuição espacial: circuito e polo.

Entende-se circuito espacial de produção da LT como uma região extensa, complexa e contínua, sendo definido a partir da elevada concentração de casos em um período considerado, na maior parte das vezes, se superpondo a mais de um município, composto de áreas com diferentes gradientes de densidades de casos. Estes são decorrentes de processos socioambientais particulares e dinâmicos, podendo apresentar tendência à expansão ou retração, em função das características de seus determinantes. Já o polo é uma unidade espacial mais restrita, que se destaca por sua densidade intensa de casos em contraste com áreas vizinhas, sendo uma unidade dinâmica, pulsátil, onde os limites e a intensidade de transmissão variam momento a momento ${ }^{12,14}$. 

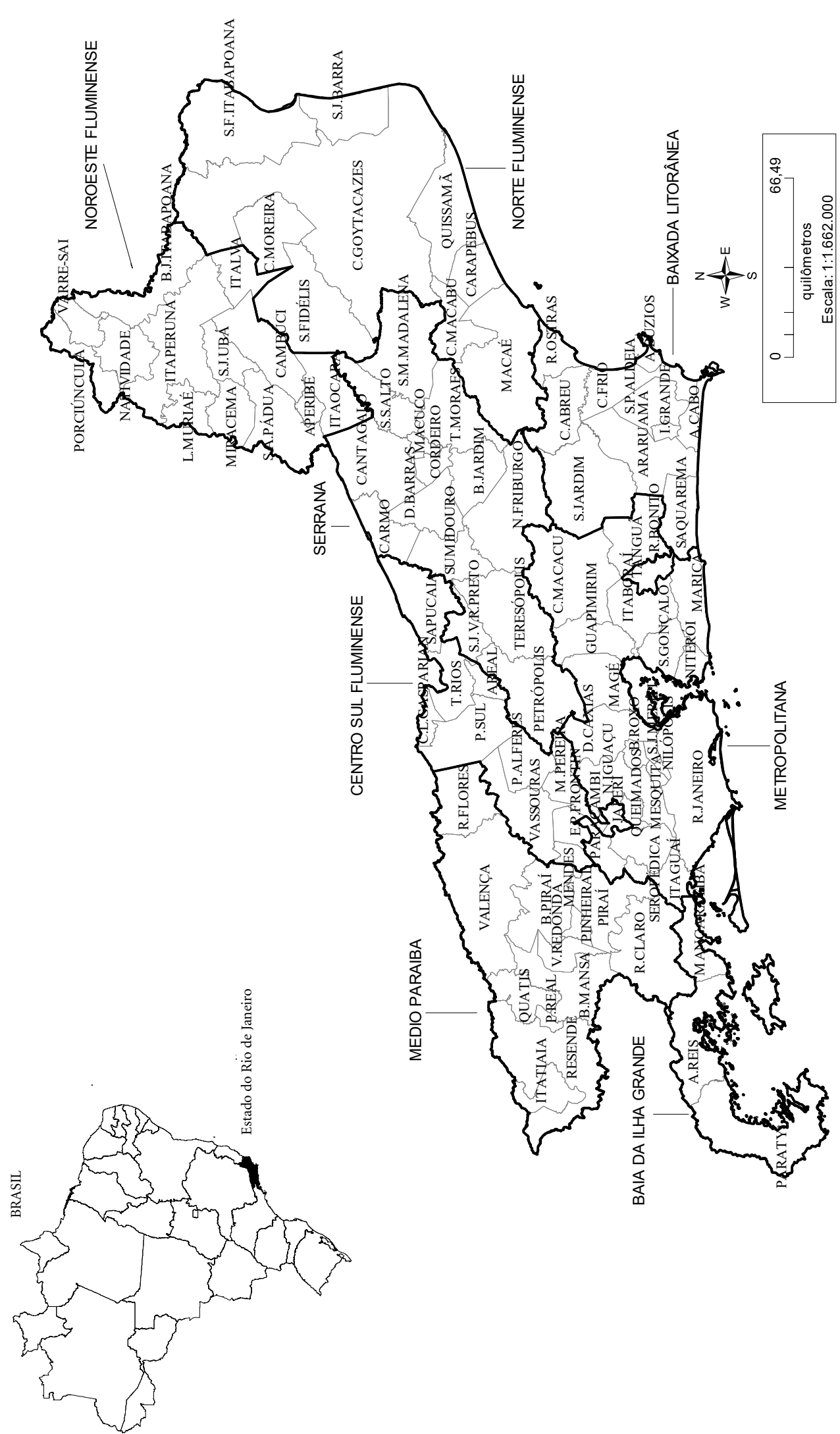

Figura 1. Estado do Rio de Janeiro por municípios e regiões de governo. Brasil. 
Diversas modalidades de polos podem ser identificadas: polos nucleares - identificados como áreas de alta densidade dentro de circuitos espaciais de LT; polos circunscritos - aqueles que se destacam de modo isolado, em regiões de baixa densidade de casos, fora de circuitos e polos emergentes - considerados como novas áreas atratoras, reconhecidas durante a comparação das densidades de casos registrados em um período, com as análises dos dados acumulados no período ante$\operatorname{rior}^{12,14}$.

A fim de destacar toda região do estado, que em algum momento da série histórica teve importância na notificação da doença, adotou-se uma terceira categoria, a de circuitos e polos resultantes, obtidos com a sobreposição dos contornos dos circuitos e polos de cada período, redesenhando um novo contorno resultante de toda a série ${ }^{15}$.

Foi utilizada a densidade de casos de LT por período para a análise da distribuição espacial da endemia, ou seja, a média dos números de casos do município por período, dividido pela área em $\mathrm{Km}^{2}$ do município, e multiplicado por $1.000^{13}$.

Para cada período, os polos e os circuitos mais relevantes de ocorrência da LT foram identificados por meio do interpolador inverso da distância (Inverse Distance Weighting - IDW) do indicador densidade de casos de LT. Este interpolador calcula o valor para cada uma das células da grade que cobre a área mapeada. O método de interpolação considera o peso inverso à distância, ou seja, quanto mais distante o ponto (nesse caso, o centroide do município com o valor do indicador "densidade de casos de LT") estiver da célula, menor será a influência do seu valor. O aplicativo utilizado foi o MAPINFO 11.0. Para esta operação foram definidas células do tamanho de $1,5 \mathrm{~km}$, um valor exponencial de 2 e uma largura de banda de $37 \mathrm{~km}^{16}$.

Para a elaboração dos mapas temáticos considerou-se a divisão por município de 2010. Para o indicador incremento populacional a população adotada foi a dos Censos do IBGE de 1991 e 2010 e para densidade populacional o Censo de $2010 \mathrm{e}$ área em $\mathrm{Km}^{2}$. Aplicou-se também o interpolador inverso da distância dos dados através do software MAPINFO 11.0. Foram utilizadas células do tamanho de $2 \mathrm{~km}$, um valor exponencial de 2 e uma largura de banda de $40 \mathrm{~km}$.

Foram empregadas duas imagens do estado com informações sobre cotas de altitude (Shuttle Radar Topography Mission-SRTM), disponível no site da Embrapa $^{17}$ e outra imagem do INEA sobre cobertura vegetal e uso da terra ${ }^{18}$. A estes mapas foi sobreposta uma camada georreferenciada do contorno resultante dos circuitos e polos, para auxiliar a caracterização das áreas.

Este estudo foi aprovado no Comitê de Ética em Pesquisa da Escola Nacional de Saúde Pública Sérgio Arouca da Fundação Oswaldo Cruz.

\section{Resultados}

No período de estudo, foram registrados 5.263 casos de LT no estado do RJ, onde observou-se estabilidade na ocorrência anual em torno de 260 notificações até 2006, com destaque para os anos de 1993 e 1994, com 497 e 388 ocorrências respectivamente. A partir de 2006 verificou-se queda importante na notificação do agravo, perdurando até o final do estudo. Importantes variações foram percebidas em determinados momentos, possibilitando o reconhecimento de um comportamento cíclico na série histórica, com ocorrência de aumentos na detecção de casos em períodos de dois a três anos (Figura 2).

Os mapas do indicador de densidade de casos de LT nesses períodos mostraram a presença do agravo em quase todo estado. O método utilizado permitiu delimitar as áreas onde foram registradas as maiores concentrações deste indicador nos chamados circuitos e polos (Figura 3).

No primeiro período (1990 - 1992), a maior ocorrência dos casos foi verificada no município de Parati e em alguns da região Metropolitana do RJ, como Mangaratiba, Itaguaí, Rio de Janeiro e Magé. Notou-se neste período ocorrências, de menor intensidade, nos municípios de Trajano de Morais, Santa Maria Madalena, Porciúncula e Maricá.

O segundo período (1993 - 1995) se caracterizou pelo aumento expressivo do número de casos em todo estado e aumento da sua área de ocorrência. Observou-se a presença de um circuito de produção da doença (circuito1), atingindo a região Metropolitana e a região da Baía da Ilha Grande (Angra dos Reis e Parati), onde três principais polos nucleares tiveram relevância, um em Parati, um em Itaguaí e outro no Rio de Janeiro. No leste da região serrana, o circuito 2 , se evidenciou um polo nuclear no município de Trajano de Morais. No oeste da região Serrana, sobressaiu o polo circunscrito 2 que compreendeu os municípios de Três Rios e São José do Vale do Rio Preto. Outro polo circunscrito foi identificado em Rio Bonito (Polo circunscrito 1). Se manteve o polo emergente 4 de Porciúncula e um novo polo no município de Cambuci (Polo emergente 2). 


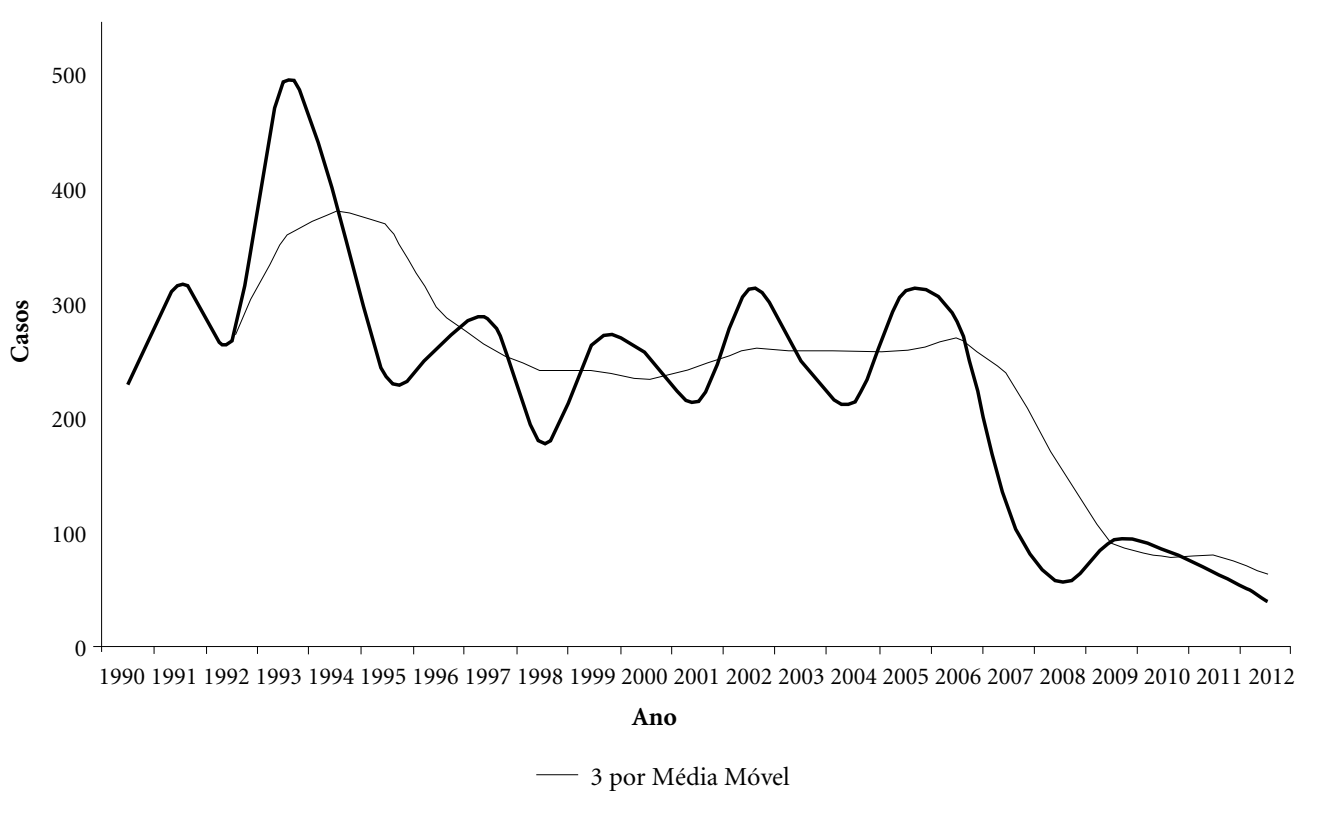

Figura 2. Distribuição dos casos de LT no estado do Rio de Janeiro, de 1990 a 2012. Brasil.

No terceiro período (1996 - 1998), o circuito 2 apresentou redução das notificações, permanecendo o circuito 1 como principal região de produção da doença, ainda que com menor intensidade, mantendo como principais polos nucleares os municípios do RJ, Itaguaí e Parati.

No quarto período (1999 - 2001), o circuito 1 se fragmentou em dois polos nucleares de maior importância, um na região da Baia da Ilha Grande (Parati e Angra dos Reis) e outro no município do Rio de Janeiro, ambos com menor intensidade que no período anterior. $\mathrm{O}$ circuito 2 , apesar de ter mantido mais baixa notificação de casos que nos dois períodos anteriores, teve o polo nuclear deslocado para Bom Jardim e Cordeiro. O importante polo circunscrito 2 ressurgiu no oeste da região Serrana, em Sapucaia e São José do Vale do Rio Preto e outro, de menor intensidade, em Saquarema (Polo circunscrito 1), na região dos Lagos. Um polo emergente foi detectado no município de Varre-Sai (Polo emergente 2), no norte do estado.

No quinto período (2002-2004) permaneceram os polos circunscritos de Saquarema, na região dos Lagos e os municípios de Sapucaia e Comendador Levy Gasparian, na região Serrana. $\mathrm{O}$ circuito 2 e os polos da região Noroeste e Norte perderam importância. Neste período houve recomposição do circuito 1 com um polo nuclear no município do Rio de Janeiro e o outro em Angra dos Reis e Parati.

No sexto período (2005 - 2007), o circuito 2 permaneceu com baixa ocorrência de casos, com um polo nuclear no município de São Sebastião do Alto. O polo circunscrito 1, de pequena intensidade, se manteve nos municípios de Saquarema e Maricá. Novamente o circuito 1 se fragmentou, com apenas um polo nuclear de maior importância no município do Rio de Janeiro.

Os períodos sete (2008-2010) e oito (20112012) se caracterizaram pelo baixo registro de casos em todo estado. Ressaltou-se a importante retração do circuito 1 , apresentando apenas polos nucleares de alguma importância nos municípios do Rio de Janeiro e Japeri. O polo circunscrito 1 , com baixa ocorrência de casos, se manteve no último período no município de Saquarema.

Dos 92 municípios do estado (Figura 4), 37 tiveram importância no registro da LT e se encontravam dentro dos circuitos e polos resultantes. São eles:

- Circuito 1 (20 municípios) - Angra dos Reis, Belford Roxo, Duque de Caxias, Itaguaí, Japeri, Magé, Mangaratiba, Mendes, Mesquita, 


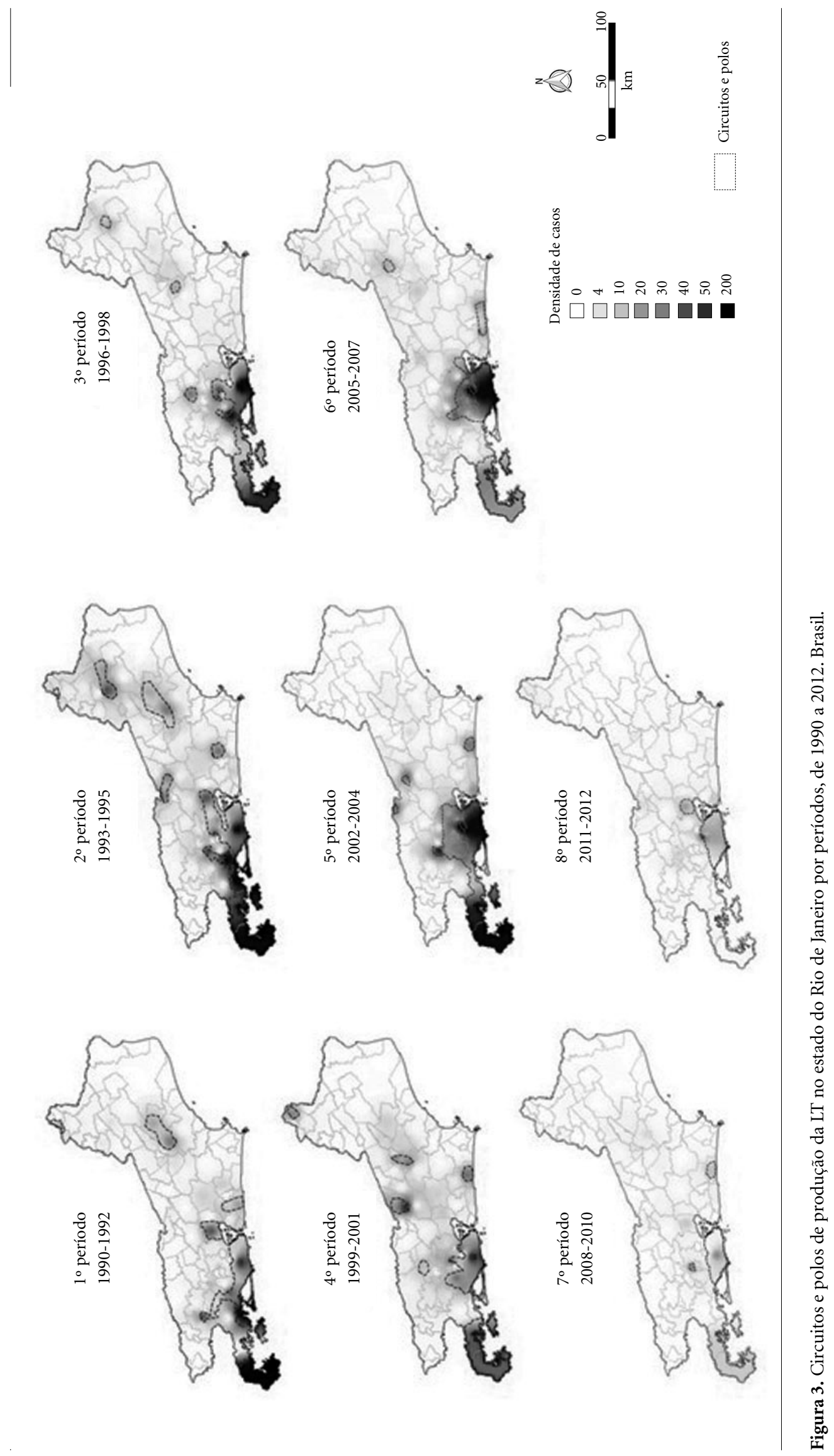


Figura 4. Circuitos e polos resultantes de LT no estado do Rio de Janeiro no período de 1990 a 2012. Brasil.

Nilópolis, Nova Iguaçu, Paracambi, Parati, Pirai, Queimados, Rio de Janeiro, São João de Meriti, Seropédica, Vassouras e Volta Redonda.

- Circuito 2 (5 municípios) - Bom Jardim, Cordeiro, São Sebastião do Alto, Santa Maria Madalena e Trajano de Morais.

- Polo circunscrito 1 (4 municípios) - Itaboraí, Maricá, Saquarema e Rio Bonito.

- Polo circunscrito 2 (4 municípios) - Comendador Levy Gasparian, Três Rios, São José do Vale do Rio Preto e Sapucaia.

- Polo emergente 3 (2 municípios) - Cambuci e Italva.

- Polo emergente 4 (2 municípios) - Porciúncula e Varre-Sai.

Dos 5.263 casos da doença notificados no período, $86,51 \%$ destes (4.552), ocorreram nos municípios localizados nestes circuitos e nos polos resultantes. O circuito 1 registrou 3.652 casos $(80,23 \%)$, no circuito 2 ocorreram 373 novos casos $(8,19 \%)$, nos polos circunscritos 1 e 2 os casos detectados foram 229 e 154, correspondendo a $5,03 \%$ e $3,38 \%$ respectivamente e nos polos emergentes 3 e 4, as notificações foram 101 $(2,29 \%)$ e 43 casos, que corresponde a $0,94 \%$ do total de casos verificados.

Ao analisar os dados do banco SINAN de 2000 a 2012, observou-se pequenas variações entre os circuitos e os polos resultantes para os indicadores epidemiológicos, com 58,9\% em média das notificações em pessoas do sexo masculino e expressiva ocorrência em crianças menores de 10 anos, em média de 8,6\%.

Os indicadores de serviço mostraram que cerca de $20 \%$ dos diagnósticos foram realizados de forma clínico-epidemiológica e que quase metade das pessoas com cura clínica não foram relatadas no sistema. Importante variação, no entanto, foi detectada nas informações, quanto ao percentual de forma mucosa entre os circuitos e 


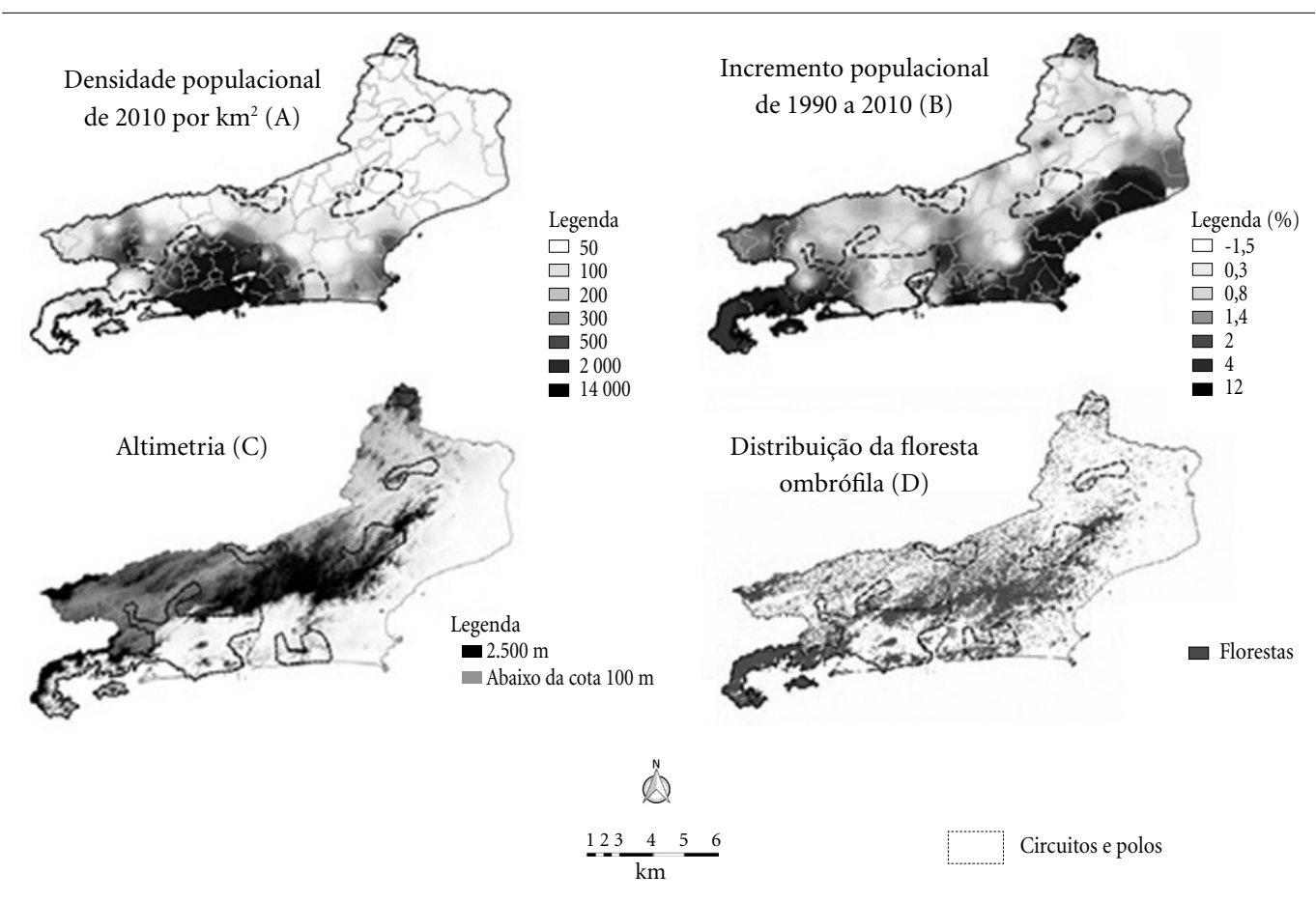

Figura 5. Distribuição dos circuitos e polos resultantes de produção de LT no estado do Rio de Janeiro de 1990 a 2012 sobrepostos as camadas de indicadores populacionais e ambientais. Brasil.

polos $(5,88$ e 18,18\%).

A Figura 5 (A) evidencia a notificação da LT tanto nas áreas de alta como nas de baixa densidade populacional, mesmo comparando polos nucleares do mesmo circuito. Observou-se uma grande concentração de casos no polo do município do Rio de Janeiro, de alta densidade populacional e naqueles de baixa densidade como no polo da região da Baía da Ilha Grande, ambos no circuito 1.

$\mathrm{Na}$ Figura 5 (B), destacou-se a presença de polos e circuitos de LT em áreas de decremento populacional, como nos municípios do circuito 2 e outros em áreas de forte atração populacional, como o polo nuclear da Baía da Ilha Grande, nos municípios de Angra dos Reis e Parati, no circuito 1 .

"A Figura 5 (C), na escala de estado, mostrou que a ocorrência da doença se deu tanto abaixo da cota $100 \mathrm{~m}$ (metros) quanto em outras áreas mais elevadas."

A Figura 5 (D) apontou polos e circuitos com grande presença de Mata Atlântica e outros polos e circuitos onde predominou variados tipos de vegetação, mas sempre com alguma cobertura vegetal, embora às vezes apenas representada por fragmentos.

\section{Discussão}

A LT, por ser uma doença focal, necessita que seu controle seja local, porém a instabilidade de um processo endêmico-epidêmico em constante movimento como este ${ }^{13}$ requer a identificação das regiões de maiores ocorrências da doença (os circuitos e os polos de produção da doença), com a finalidade de serem operadas do ponto de vista da vigilância e do controle ${ }^{19}$.

A identificação dos circuitos e polos mais estáveis que as localidades que os compõem, permitem operações de vigilância e controle não somente naquelas com muitos casos, mas em todas as localidades da área de risco identificada, por terem as mesmas características das localidades já afetadas, dependendo somente de mudanças nas condições de receptividade ${ }^{13}$.

$\mathrm{O}$ ajustamento dos dados espacialmente referenciados permitiu delimitar áreas onde foram registradas as maiores concentrações de densida- 
de de casos de LT no estado do RJ, chamadas circuitos e polos. No período observou-se a grande instabilidade dessas áreas, com grande variação na intensidade de casos, na forma, na área ocupada, em alguns casos desaparecendo em determinados períodos e reaparecendo em outros.

Apenas o circuito 1 esteve presente em todos os períodos da série histórica. Neste circuito podemos destacar dois núcleos de maior intensidade, situados um no município do Rio de Janeiro e outro na Região da Baía da Ilha Grande (Parati e Angra dos Reis), como principais polos nucleares de ocorrência da LT, evidenciando este circuito como de maior relevância para a produção da doença no estado.

De acordo com os vários estudos locais no estado, a doença tem características epidemiológicas semelhantes, tanto nas espécies de flebotomíneos ${ }^{20}$, parasitas $^{21}$ e reservatórios, envolvidos na transmissão ${ }^{22}$, como nas distribuições por sexo e grupos etários evidenciados neste estudo, indicando uma transmissão no intra e peridomicílio ${ }^{8,23}$. No entanto, importantes diferenças foram observadas quando se procurou analisar os processos de organização social, características ambientais e geográficas com as condições de endemicidade, permitindo a identificação dos diferentes circuitos e polos de produção e difusão da LT.

Estudos realizados em outros locais, dentro e fora do país, mostram a identificação dos circuitos de produção da doença. Temponi et al. ${ }^{16}$ identificaram três circuitos importantes, distribuídos nas mesorregiões Norte de Minas Gerais, Vale do Rio Doce e Região Metropolitana de Belo Horizonte e mostraram que houve forte associação dos casos de LT com lavoura temporária, pastagem natural, floresta natural, terras inaproveitáveis e população rural. Os autores relatam ainda que os resultados encontrados demonstram que nas áreas com maior transmissão da doença, identificadas pelos circuitos espaciais de produção, mostram o caráter focal da LT e também as diferenças regionais quanto à transmissão no estado no período do estudo. Rodriguéz et al. ${ }^{24}$, em estudo realizado na Venezuela, identificam três padrões de transmissão na área de pesquisa. Os autores relatam que a LT apresentava uma tendência periódica sendo observado cinco clusters espaço-temporais em toda a região.

O local de transmissão da LT é um foco com características epidemiológicas semelhantes no estado, que pode ser reproduzido em regiões diferentes, com dinâmicas distintas, tornando fundamental a identificação dessas regiões, que são os circuitos e polos. Sendo assim, fica evidente a importância dos vetores de organização regional nas situações locais de risco, criando situações de vulnerabilidades socioambientais para o recrudescimento da doença em alguns momentos e redução em outros ${ }^{11}$.

No circuito 1, por exemplo, um padrão característico de agricultura do polo da Baía da Ilha grande, eram praticados em moldes tradicionais, voltadas para a pesca, a cultura de banana e da mandioca, que proporcionavam uma receptividade com manutenção da população de vetores e reservatórios, bastando a chegada de suscetíveis para que a transmissão se amplificasse. Com a construção da estrada Rio Santos, teve início o processo de substituição deste padrão de pequenas propriedades por áreas privadas utilizadas principalmente para o lazer e especulação imobiliária, proporcionando uma evasão de população do campo em direção as sedes dos municí$\operatorname{pios}^{13,25}$. Isto certamente favoreceu a diminuição da receptividade das áreas à $\mathrm{LT}$, com queda das populações de vetores e reservatórios no ecossistema modificado, levando a uma importante redução das notificações de casos da doença, observada principalmente a partir de $2006^{13}$.

Nos municípios do circuito 2, a consolidação da ocupação desta porção do território fluminense foi devido principalmente à cultura cafeeira. Com o declínio desta cultura, as atividades agropecuárias extensivas que a substituíram não conseguiram evitar o esvaziamento econômico e demográfico que se seguiu, provocando uma diminuição nos efetivos populacionais, principalmente da zona rural ${ }^{25}$.

Diante dessa situação, com base na observação de campo, o autor principal deste artigo verificou que com o objetivo de evitar a perda de receita com o fundo de participação dos municípios, os prefeitos da região ofereciam pequenos lotes para fixação dessas famílias nas periferias das sedes. As famílias, com intuito de melhorar a renda, realizavam plantio intensivo de banana nos terrenos e construíam suas residências no interior das plantações, criando assim condições epidemiológicas que podem favorecer a transmissão da LT. A implantação da lei orgânica dos municípios, em meados da década de $1990^{26,27}$, contribuiu para o processo de urbanização, trazendo desenvolvimento dos setores econômicos. Essa situação levou a uma redução da receptividade à LT nas periferias das sedes municipais, contribuindo para a queda da transmissão da doença, principalmente a partir de 2006.

Houve redução da receptividade nessas regiões a partir de 2006, no entanto, nos anos seguin- 
tes à 2012, observou-se somente o registro de casos esporádicos da doença dispersos nos municípios do estado, indicando a permanência da estrutura epidemiológica adequada à transmissão, embora não sendo relevante na delimitação da área de risco para $\mathrm{LT}^{13,28}$. A redução no número de casos da doença foi observada também por Amaro \& Costa em 2017 na Região Metropolitana do Rio de Janeiro ${ }^{29}$.

A transitoriedade é uma característica do processo endêmico-epidêmico no nível local, mesmo na ausência de controle efetivo, acarretado por mudanças de receptividade no território. Uma questão fundamental para subsidiar as ações de vigilância e controle ambiental da leishmaniose tegumentar, deve ser a importância da compreensão do processo de transmissão em nível local discutindo o desenvolvimento de uma metodologia capaz de identificar um padrão de ocorrência da doença, e contribuir para o conhecimento da dinâmica e circulação do parasita entre os focos de uma mesma região de alta transmissão ${ }^{13}$.

Diante disso, a identificação e a caracterização dessas unidades espaciais (circuitos e polos) ${ }^{16}$ pode ter a finalidade de subsidiar o programa estadual para a priorização de estratégias de ações voltadas para prevenção, controle e otimização dos recursos do programa.

\section{Colaboradores}

VB Soares, A Sobral e PC Sabroza: concepção, planejamento, análise e interpretação dos dados, revisão e aprovação da versão final do manuscrito. WP Vargas, R Souza-Santos e ACO Valdés: análise dos dados, revisão e aprovação da versão final do manuscrito. 


\section{Referências}

1. Santos M. Espaço Dividido. 2a ed. São Paulo: Editora da Universidade de São Paulo; 2008.

2. Santos M. Manual de Geografia Urbana. 3a ed. São Paulo: Editora da Universidade de São Paulo; 2008.

3. Sabroza PC, Kawa H, Campos WSQ. Doenças transmissíveis: ainda um desafio. In: Minayo MCS, organizadora. Os muitos Brasis: saúde e população na década de 80. São Paulo: Hucitec; 1995. p.177-244.

4. Sabroza PC. O domicílio como fator de risco na Leishmaniose Tegumentar Americana: estudo epidemiológico em Jacarepaguá, município do Rio de Janeiro [tese]. Rio de Janeiro: Fiocruz; 1981.

5. Sabroza PC, Toledo LM, Osanai CH. A organização do espaço e os processos endêmico-epidêmicos. In: Leal MC, Sabroza PC, Rodriguez RH, Buss PM, organizadores. Saúde, ambiente e desenvolvimento. São Paulo: Hucitec; Abrasco; 1992. p. 57-77.

6. Negrão GN, Ferreira MENC. Considerações sobre a dispersão da leishmaniose tegumentar americana nas Américas. Rev Percurso 2009; 1(1):85-103.

7. Silva LMR, Cunha PR. A urbanização da leishmaniose tegumentar americana no municípiode Campinas - São Paulo (SP) e região: magnitude do problema e desafios. An Bras Dermatol 2007; 82(6):515-519.

8. Valim C. Transmissão da Leishmania (Viannia) brasiliensis no Ceará [tese]. Rio de Janeiro: Fiocruz; 1993.

9. Brasil. Ministério da Saúde (MS). Manual de Controle da Leishmaniose Tegumentar Americana. Brasília: MS; 2000.

10. Menezes CR, Azevedo AC, Costa SM, Costa WA, Rangel EF. Ecology of American cutaneous leishmaniasis in the state of Rio de Janeiro. Brazil J Vector Ecol 2002; 27(2):207-214

11. Kawa H, Sabroza PC, Oliveira RM, Barcellos C. A produção do lugar de transmissão da leishmaniose tegumentar: o caso da Localidade Pau da Fome na cidade do Rio de Janeiro, Brasil. Cad Saude Publica 2010; 26(8):1495-1507.

12. Soares VB, Sabroza PC, Kawa H. 1995. Padrões de transmissão da leishmaniose tegumentar no estado do Rio de Janeiro. In: III Congresso Brasileiro, II Congresso Ibero-Americano, I Congresso Latino Americano de Epidemiologia, Anais, p. 282. Salvador: Abrasco.

13. Soares VB, Almeida AS, Sabroza PC, Vargas WP. Vigilância epidemiológica da Leishmaniose tegumentar: análise territorial local. Rev Saude Publica 2017; 51:51.

14. Brasil. Ministério da Saúde (MS). Manual de Vigilância da Leishmaniose Tegumentar Americana. 2a ed. Brasília: Editora do Ministério da Saúde; 2007. (Série A. Normas e Manuais Técnicos)

15. Soares VB. Proposta de vigilância epidemiológica da leishmaniose tegumentar em nível local: análise de indicadores para região endêmica da mata atlântica no estado do Rio de Janeiro de1990 a 2004 [dissertação]. Rio de Janeiro: Fiocruz; 2006.

16. Temponi AOD, Brito MG, Ferraz ML, Diniz SA, Cunha TN, Silva MX. Ocorrência de casos de leishmaniose tegumentar americana: uma análise multivariada dos circuitos espaciais de produção, Minas Gerais, Brasil, 2007 a 2011. Cad Saude Publica 2018; 34(2):e00165716.

17. Brasileira de Pesquisa Agropecuária (Embrapa). Brasil em relevo - Rio de Janeiro. [acessado 2010 Out 15]. Disponível em: https://www.cnpm.embrapa.br/projetos/relevobr/download/rj/ri.htm
18. Instituto Estadual do Ambiente (INEA). Cobertura vegetal e uso da terra. [acessado 2010 Out 15]. Disponível em: http://200.20.53.3:8081/Portal/MegaDropDown/Monitoramento/Coberturavegetaleusodaterra/index.htm\&lang

19. Negrão GN, Ferreira MEMC. Considerações sobre a Leishmaniose Tegumentar Americana e sua Expansão no Território Brasileiro. Revista Percurso 2014; 6(1):147-168.

20. Barbosa Santos MEG, Marzochi MCA, Massard CL, Lima GPS, Confort EM. Aspectos Epidemiológicos da leishmaniose tegumentar em cães, no município de Paraty, Estado do Rio de Janeiro, Brasil. Cad Saude Publica 1999; 15(3):641-646.

21. Gontijo CM, Silva ES, Fuccio MB, Souza MC, Pacheco RS, Dias ES, Andrade Filho JD, Brazil RP, Melo MN. Epidemiological studies of an outbreak of cutaneous leishmaniasis in the Rio Jequitinhonha Valley, Minas Gerais, Brazil. Acta Trop 2002; 81(2):143-150.

22. Kawa H And Sabroza PC. JunEspacialização da leishmaniose tegumentar na cidade do Rio de Janeiro. Cad Saude Publica 2002; 18(3):853-865.

23. Brasil. Ministério da Saúde (MS). Manual de vigilância da leishmaniose tegumentar. $2^{\text {a }}$ ed. Brasília: MS; 2017. [acessado 2018 Jan 24]. Disponível em: http:// bvsms.saude.gov.br/bvs/publicacoes/manual_vigilancia_leishmaniose_tegumentar.pdf

24. Rodríguez EM, Díaz F, Pérez MV. Spatio-temporal clustering of American cutaneous leishmaniasis in a rural municipality of Venezuela. Epidemics 2013; 5(1):11-19.

25. Centro de Estatísticas, Estudos e Pesquisas do Estado do Rio de Janeiro (Ceperj). Histórico das Regiões de Governo. [acessado 2016 Jun 28]. Disponível em: http://www.ceperj.rj.gov.br/ceep/info_territorios/divis_regional.html

26. Santa Maria Madalena. Lei Orgânica do Município de Santa Maria Madalena. Rio de Janeiro, 1990. [acessado 2016 Nov 28]. Disponível em: http://www.sepexrio.org.br/wp-content/uploads/2013/01/lei_organica_santa_maria_madalena.pdf

27. Trajano de Morais. Lei Orgânica do Município de Trajano de Morais. Rio de Janeiro, 1990. [acessado 2016 Nov 28]. Disponível em: http://camaratrajano.com. br/wp-content/uploads/2013/02/Lei-Organica-do -Municipio-de-Trajano-de-Moraes.pdf

28. Brasil. Ministério da saúde (MS). GT-Leishmanioses/ UVTV/CGDT/DEVIT. Dados sobre casos de LTA disponibilizados em 13 de julho de 2017 via email. Brasília: MS; 2017.

29. Amaro RF, Costa WA. Transformações socioespaciais no estado do Rio de Janeiro enquanto determinante social da saúde: no contexto das leishamnioses. Revista Brasileira de Geografia Médica e da Saúde. Hygeia 2017; 13(26):198-210.

Artigo apresentado em 23/04/2018

Aprovado em 11/10/2018

Versão final apresentada em 13/10/2018 
